[CONTRIBUTION FROM THE CHEMTCAI, LABORATORY OF THE UNIVERSITY OF CALIFORNIA.]

\title{
SOLUBILITY. IV. SOLUBILITY RELATIONS OF NAPHTHALENE AND IODINE IN THE VARIOUS SOLVENTS, INCLUDING A METHOD FOR EVALUATING SOLUBILITY DATA.
}

\author{
By Joer H. Hildebrand and Clarence A. Jeniss. \\ Received July 29, 1920.
}

In the preceding papers of this series ${ }^{1}$ a theory of solubility has been presented according to which Raoult's law, which is taken as expressing the behavior of an ideal solution, will be obeyed by substances which, in the liquid state, have the same internal pressures, provided the substances are relatively non-polar and do not tend to form compounds with each other. Positive deviations from Raoult's law, under the same conditions, are shown wherever the substances have different internal pressures. Since solubilities can be calculated on the basis of Raoult's law, substances will be less solible in each other the greater the difference in their internal pressures in the liquid state.

In the earlier papers the solubility relations of naphthalene and iodine were briefly discussed. It is the purpose of the present paper to do this more fully, with the aid of a new method for criticising solubility data, and utilizing additional experimental material.

\section{Method for Evaluating Solubility Data.}

In evaluating solubility data, it is possible to use a procedure irequently applied in connection with vapor pressures. From the form of the ideal equation, $\log N=L / 4.57 T+I$, where $N$ is the mol-fraction of the solute in solution, $L$ its heat of solution, and $I$ a constant of integration, it is evident that when $\log N$ is plotted against $\mathrm{r} / T$, a straight line should result whose slope gives the heat of solution of the solute (which is identical with the heat of fusion of the solute when Raoult's law is obeyed). Moreover, when the 2 substances in the liquid form are miscible in all proportions, as is the case where Raoult's law is even approximately obeyed, $N$ becomes unity at the melting point of the solute. It follows, therefore, that for solutions in which molecular changes do not play very important roles, the solubility data when plotted in the above fashion will give a family of curves converging to the melting point and approaching the ideal value calculated from the heat of fusion as Raoult's law is approached. Figs. I and 3 illustrate this method.

Since the internal pressures of different substances do not retain precisely the same relative values at all temperatures, the solubility lines are not strictly parallel. This is, however, a second order effect. It is possible, therefore, to make the following predictions for solutions of nonpolar substances.

1 This Journal, 38, 1452 (1916); 39, 2297 (I917); 41, 1067 (1919). 
I. From the melting point of the solute and its solubility at any one temperature (in terms of mol-fraction) it is possible to predict its solubility at all temperatures rather closely by drawing a straight line through the 2 points on the plot of $\log N$ against $\mathrm{I} / T$. Where the 2 substances differ greatly in internal pressure, which can readily be determined according to the criteria used in the third paper of this series, this line should be slightly concave upwards.

2. When the solubility of the solute it other solvents is known at any temperatures, a family of curves similar to those in Figs. I and 3 can be drawn. It is then possible to predict approximately its solubility in any other soivent at any temperature by tuoting the relation of the internal pressure of the new solvent to that of the others, and by drawing a line in the proper position. This will be illustrated in the subsequent discussion of existing data.

3. When solvation exists solubility will be greater than would otherwise be expected, but will have a smaller temperature coefficient.

4. The temperature coefficient of solubility will be greatest for substances differing most in internal pressure.

\section{Solubility Relations of Naphthalene.}

At the time of the publication of the first paper of this series, we were not aware of an important paper upon solubility by Schroeder ${ }^{1}$ in which he published, apparently for the first time, the formula for calculating the solubility of a substance from its heat of fusion and melting point upon the assumption that Raoult's law holds. He further represented his results by plotting $\log N$ against the difference between the melting point of naphthalene and the temperature of each solubility determination. This method is similar to the one here enployed, but does not give straight lines. The data he obtained using solutions of naphthalene in benzene, chlorobenzene and carbon tetrachloride, agree fairly well with the formula. We wish, however, to take account of the minor deviations of solubility in these and other solvents and to show that they are essentially such as have been predicted on the basis of internal pressure. In addition to the data of Schroeder, we have determinations of the solubility of naphthalene in hexane, carbon disulfide and chloroform by Etard ${ }^{2}$ and by Speyers ${ }^{3}$ in chloroform and toluene. All of the above determinations cover a considerable range of temperature. The data of Etard and Speyers for chloroform agree fairly well. A single determination by Bechi in toluene agrees with the values by Speyers.

The experimental values have been plotted in Fig. I. The ideal solu-

${ }^{1}$ Schroeder, $Z$. physik. Chem., II, 449 (I893).

2 E,tard, Bull. soc. chim., [3] 9, 82 (1893).

${ }^{3}$ Speyers, Am. J. Sci., [4] 14, 294 (1902).

'Bechi, Ber, 52,1978 (1879). 
bility calculated from the heat of fusion is also indicated. For this purpose, the heat of fusion of naphthalene and its specific heats in the solic and liquid forms have been taken from the work of Bogojawlenski. ${ }^{1}$ His value of the heat of fusion is a little lower than the ones given by older observers, but the comprehensive character of the investigation and the greater purity of the naphthalene used, as indicated by its higher melting point, are evidence that this work is the most accurate. If other values were taken into account the line would be slightly higher, but the validity of the theory would not be affected thereby.

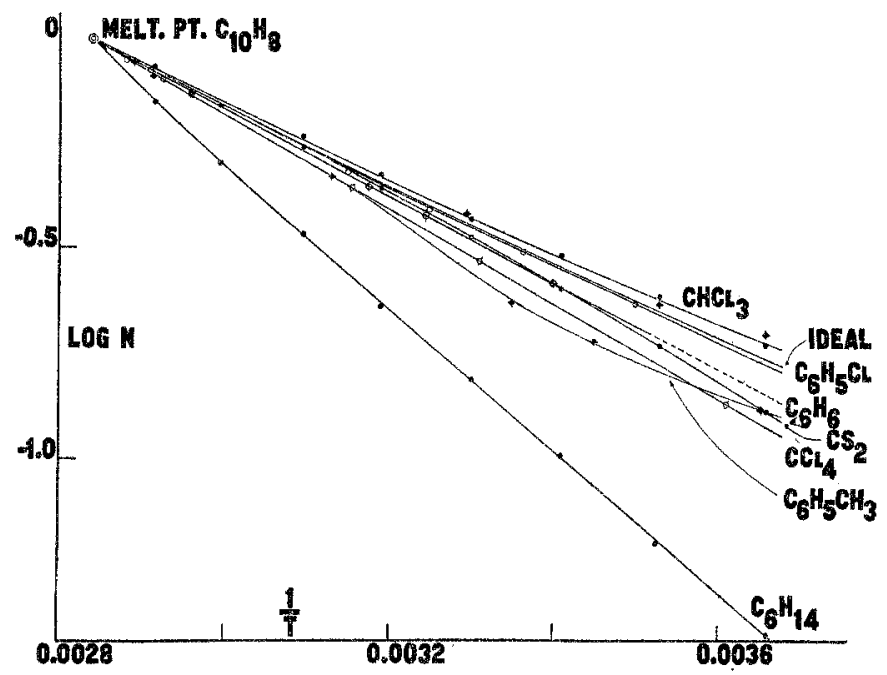

Fig, 1,-Solubilities of naphthalene.

It will be seen that, except for chloroform and toluene, the solvent powers of the various substances considered are exactly in the order given in the table of relative internal pressures in the third paper of this series, where the values are shown to increase in the following order: hexane, carbon tetrachloride, toluene, benzene, chloroform, chlorobenzene, naphthalene, carbon disulfide. We would expect from this that the solubility of naphthalene in chloroform would be between its solubilities in benzene and chlorobenzene. 'The fact that its solubility in this solvent is greater than Raoult's law would lead us to expect, may be evidence of the existence of solvation such as will be seen in the case of iodine in benzene, and, at lower temperatures, in carbon disulfide.

The curve for toluene should, theoretically, fall slightly above that for carbon tetrachloride, and be almost straight, like those for the other solvents. The fact that it falls below for part of its course would constitute a. more serious contradiction if the experimental values were more regular.

${ }^{1}$ Bogojawlenski, Chem. Zentr., [5] 9, II, 945 (x9o5). 
It is unlikely that the true curve would have the inflections of the one drawn in the figure, which was drawn through the experimental points. If an approximately straight line were drawn to satisfy these points it would fall very close to the one for carbon tetrachloride.

It is evident that, except for unforeseen instances involving solvation, the solubility of naphthalene in other solvents could be approximately predicted by the aid of Fig. I. For example, ethylene bromide, which falls between carbon disulfide and naphthalene, should have a solvent power somewhat greater than that of carbon disulfide, but less than the idea1. Again, xylene, which has an internal pressure between those of toluene and hexane, should show a solvent power which would be indicated by drawing a line somewhat below the line for toluene, in Fig. $\mathrm{I}$.

TABLE I.

Solubilities of Naphthalene-Mol-Fraction.

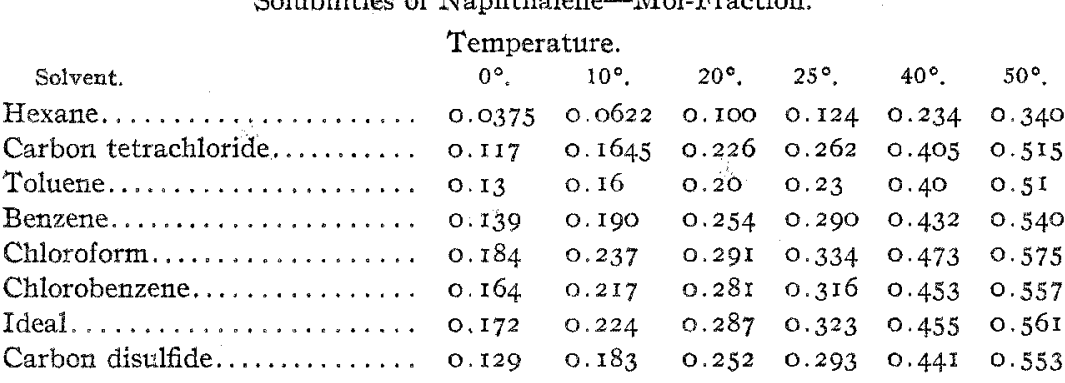

In Table I are given values of the solubilities of naphthalene at various temperatures in terms of mol-fraction. These values were taken from a careful plot of Fig. I and represent the smoothed out experimental data. The values for benzene at the lower temperatures represent some extrapolation, shown in the figure by the dotted line. This table illustrates numerically the conformity of the solubilities of naphthalene to the internal pressure theory.

\section{Solubility of Iodine.}

Experimental.-Solubilities of iodine over any range of temperature are given in the literature for only a few solvents. It was accordingly necessary for our purpose to determine the solubility in several others, including benzene.

The iodine used was subjected to a purification to eliminate particulariy the other halogens and cyanogen. For this purpose we used with but slight modification the method of Baxter. ${ }^{1}$

Commercial "C. P." benzene was first distilled and then shaken repeatedly with sulfuric acid, in order to remove thiophene. It was then frozen 4 times. The samples from the last 2 operations showed no differ-

1 Baxter, Proc. Am. Acad, 40, 42 I (1904). 
ences in freezing point greater than $0.001^{\circ}$. The benzene was finally dried with phosphorus pentoxide and redistilled.

The carbon tetrachloride used was first shaken with a concentrated solution of sodium hydroxide and then distilled from the phosphorus pentoxide, a constant-boiling distillate being obtained.

The heptane was obtained from material kindly donated by Mr. D. F. Fryer, Santa Rosa, Calif. It was treated 3 times with sulfuric acid, diluted with $1 / 2$ of its volume of water. It was then distilled, nearly all coming over between $98.3^{\circ}$ and $98.6^{\circ}$. The distillate was again shaken 3 times with sulfuric acid diluted with $1 / 4$ of its volume of water. The sulfuric acid used in this treatment remained colorless. It was then shaken with conc. sulfuric acid, treated with phosphorus pentoxide and distilled, all distilling at $98.4^{\circ}$. The density of the resulting liquid was 0.6827 at $20 . I^{\circ}$. Both the boiling point and the density agree well with the values given by other observers for normal heptane. The boiling points of the other heptanes are $90^{\circ}$ or lower.

The solubility determinations were made in an apparatus shown in Fig. 2. The bulb was supported in the thermostat in a nearly horizontal position and rotated in such a way as to rock the contained liquid from

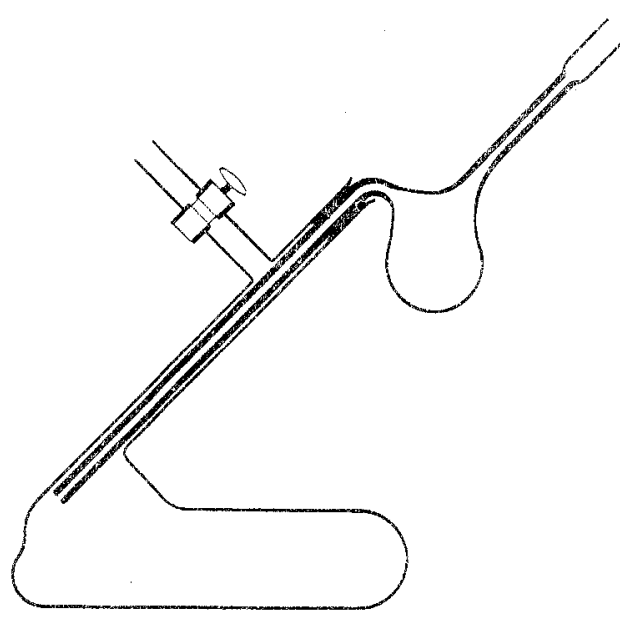

Fig. 2. one end to the other. After equilibrium was obtained the apparatus was tilted so that the exit tube was in a vertical position and a portion of the solution was forced up into the pipet by blowing gently into the side arm provided with a stopcock. The construction of the bulb is such that the solid is kept away from the tip of the pipet, minimizing the danger of forcing particles of the solid into the pipet. The bulb was kept under the surface of the water in the thermostat during the filling of the pipet. The pipet was then removed from the bulb and weighed. Its contents were washed into a flask and titrated with sodium thiosulfate solution. The weights and burets used were carefully standardized.

\section{Results.}

Tables II, III and IV give the experimental results upon the solubilities of iodine in benzene, carbon tetrachloride and heptane, respectively. These results are plotted in Fig. 3, along with the experimental values 
obtained by Arctowski for the solubility of iodine in carbon disulfide and in benzene ${ }^{2}$ at lower temperatures. The figure includes also solubilities in chloroform obtained by Hantzsch and Vagt ${ }^{3}$ and the single value of Jakowkin ${ }^{4}$ in carbon tetrachloride at $25^{\circ}$, which falls almost exactly upon our own curve. There exist values by Bruner ${ }^{5}$ for the solubilities of lodine in chloroform and carbon tetrachloride in the neighborhood of I $5^{\circ}$, but examination of his paper shows his values to be rather unreliable, and they are not here included.

TABLE II,

Solubility of Iodine in Benzene.

Temp. $25^{\circ} . \quad 30^{\circ} .35^{\circ} .40^{\circ} .45^{\circ} . \quad 50^{\circ} . \quad 54.64^{\circ}$.

G. $I_{2}$ per roo g. solu-

\begin{tabular}{|c|c|c|c|c|c|c|c|}
\hline \multirow[t]{3}{*}{$\operatorname{tion} . . . . . . . . . .}$. & 14.00 & I6.06 & 17.93 & $20.0 \mathrm{I}$ & 22.74 & $25.5 \mathrm{I}$ & 28.25 \\
\hline & 14.15 & I6. I4 & 17.74 & 20.09 & 22.82 & $\ldots$ & 28.27 \\
\hline & 14.12 & $\cdots$ & 18.03 & $\ldots$ & 22.79 & $\ldots$ & $\ldots$ \\
\hline & & & .77 .90 & .05 & 22.7 & $5.5 \mathrm{I}$ & 28.26 \\
\hline
\end{tabular}

TABLE III.

Solubility of Iodine in Carbon Tetrachloride.

Temp. $0^{\circ}$. $35^{\circ}$

G. I $I_{2}$ per Ioo g. solution.............. $0.6880 \quad 2.62 \mathrm{I} \quad 4.364$

$0.6875 \quad 2.599 \quad 4.344$

$0.6866 \quad 2.597 \quad 4.346$

\begin{tabular}{|c|c|c|c|}
\hline & . & 2.594 & . \\
\hline$\ldots \ldots \ldots$ & 0.6877 & 2.603 & $4.35 \mathrm{I}$ \\
\hline ol-fraction... & 0.00418 & $0.0 \times 594$ & 0.0268 \\
\hline
\end{tabular}

TABLE IV.

Solubility of Iodine in Heptane.

\begin{tabular}{|c|c|c|c|c|}
\hline \multirow{6}{*}{$\begin{array}{l}\text { Temp. } \\
G, I_{2} \text { per roo g. solution..... }\end{array}$} & & & & \\
\hline & $0^{\circ}$ & $25^{\circ}$ & $35^{\circ}$. & $50^{\circ}$. \\
\hline & 0.6192 & I. 703 & 2.501 & 4.200 \\
\hline & 0.6149 & $1.70 I$ & 2.497 & $4 \cdot 192$ \\
\hline & 0.6164 & $\ldots$ & 2.476 & $\ldots$ \\
\hline & 0.6199 & $\ldots$ & $\ldots$ & $\ldots$ \\
\hline Mean & 0.6176 & I. 702 & $2.49 \pi$ & $4 \cdot \times 96$ \\
\hline Mol-fraction. . & 0.00245 & 0.006786 & 0.00998 & 0.01699 \\
\hline
\end{tabular}

Marden and Dover ${ }^{6}$ have published a single determination of the solubility of iodine in chloroform at $25^{\circ}$ which does not agree very well with

${ }^{1}$ Arctowski, Z. anorg. Chem., 6, 404 (1894).

${ }^{2}$ Ibid., II, 276 (1895).

${ }^{3}$ Hantzsch and Vagt, $Z$. physik. Chem., 38, 728 (rgor).

${ }^{4}$ Jakowkin, ibid., 18, 590 (1895).

sBruner, ibid., 26, 145 (1898).

${ }^{\circ}$ Marden and Dover, This Journal, 38, I235 (I9I6). 
the values of Hantzsch and Vagt. Hertz and Rathmann ${ }^{1}$ give solubilities of iodine in chloroform at $25^{\circ}$ in terms of weight of iodine per unit volume of the solutions. Lack of data concerning the densities of these solutions makes it impossible to recalculate solubility to a weight basis. The solubility of iodine in molten sulfur is given by the freezing-point determinations of Smith and Carson. ${ }^{2}$ The formula $\mathrm{S}_{8}$ was used in calculating the mol-fraction of iodine.

\section{TABLE V.}

Solubilities of Iodine--Mol-Fraction.

Heptane.............. $0.00245 \quad 0.00376 \quad 0.005650 .00679 \quad 0.0119 \quad 0.0170$

$\begin{array}{lllllllll}\text { Carbon tetrachloride........ } & 0.00418 & 0.00631 & 0.00920 & 0.0110 & 0.0169 & 0.0268\end{array}$

Chloroform............. $0.0093 \quad 0.001350 .00193 \quad 0.0228 \quad(0.0380)(0.0530)$

$\begin{array}{llllllll}\text { Benzene.......................... } 0.0306 & 0.0420 & 0.0482 & 0.0718 & 0.095\end{array}$

$\begin{array}{lllllll}\text { Carbon disulfide........... } & 0.0250 & 0.0341 & 0.0487 & 0.0570 & 0.0925 & 0.127\end{array}$

Table V gives the smoothed-out values of the solubility of iodine at various temperatures in carbon disulfide, benzene, chloroform, carbon tetrachloride, and heptane, obtained from a careful plot of the data after the manner shown in Fig. 3 .

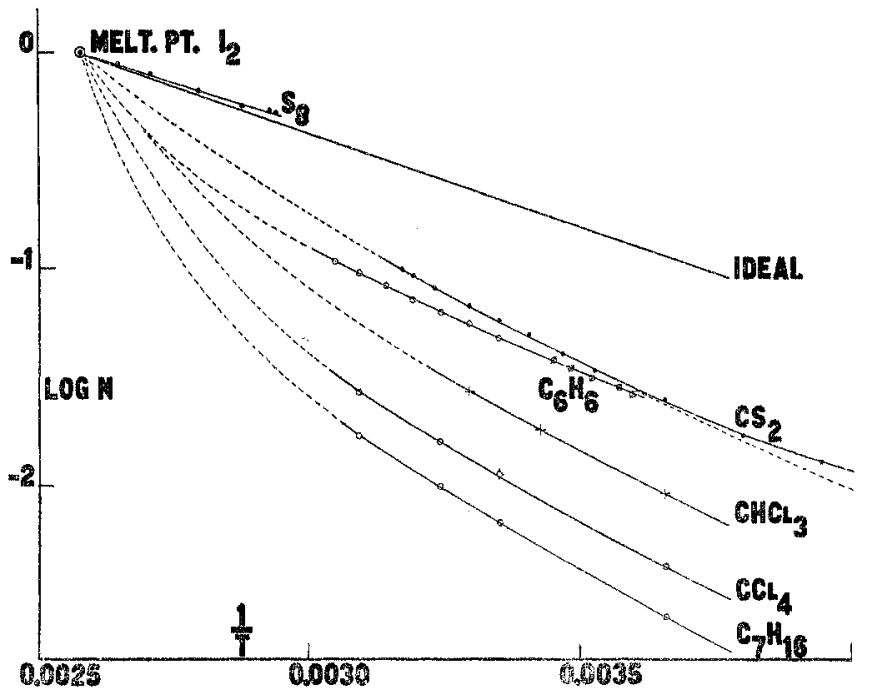

Fig. 3.-Solubilities of iodine.

Discussion.-It is evident that the solubilities of iodine plotted as in Fig. 3, give, except for benzene, a family of closely parallel curves similar to those for naphthalene shown in Fig. I. The dotted lines represent the hypothetical portions at higher temperatures as the curves converge

1 Hertz and Rathmann, Z. Elektrochem., I9, 887 (1913).

2 Smith and Carson, Z. physik. Chem, 6r, 200 (1907). 
to the melting point of iodine where it becomes soluble in all proportions, $\therefore$..,$N=1$.

Excepting for benzene, the order of solubility in the various solvents follows the order of internal pressures. Sulfur, whose internal pressure and solubility relations will be discussed more at length in a future communication, falls very close to iodine, and, since there is little or no tendency for the elements to combine, dissolves iodine almost exactly to the extent corresponding to an ideal solution.

In the first papers of this series, figures were given which showed that the solubility of iodine, in accord with the theory, falls off in the following solvents in the order named: bromoform, carbon disulfide, chloroform, carbon tetrachloride, hexane. The solubility in benzene, however, was found to be nearly as great as in carbon disulfide, instead of being near that in chloroform, as might be expected. A statement was made in the first paper attributing this discrepancy to the formation of a solid solution of iodine and benzene, reported by Beckmann and Stock. ${ }^{1}$ Prof. $E_{\text {. }}$ W. Washburn kindly directed our attention some time ago to the fact that this was a mis-statement, for it is evident that the formation of a solid solution wotld not increase solubility, but rather decrease it. Upon second thought, it seemed that the true explanation of the discrepancy lies in the solvation of iodine in benzene as shown by the fact that this solution has a brownish-red color instead of the violet shown by solutions in the other solvents above named.

Evidence that brown solutions of iodine contain solvated iodine, has been given by Hildebrand and Glasscock. ${ }^{2}$ If a solution of iodine in benzene is cooled, the red color deepens, while if it is warmed, the color approaches the violet of iodine vapor, indicating that the solvation decreases with rising temperature, as would be expected. This changing solvation with the temperature is very strikingly shown by solutions in anyl acetate, where the color may be made to vary all the way from violet to brown. When solvation occurs, the activity of the solute is diminished, allowing more of it to go into solution than would otherwise be the case, so that as the temperature rises and solvation decreases, the solubility should approach that expected from the position of the solvent in the table of internal pressures.

The effect of temperature upon the solubility of iodine in benzene is as expected from this point of view. Fig. 3 shows that as the temperature is increased the solubility in benzene approaches the solubility in chloroform. There is little doubt, therefore, that if no solvation occurred

1 Beckmann and Stock, Z. physik. Chem., I7, 107 (1895).

2 Hildebrand and Glasscock, Thrs Journal, 3I, 26 (1909). See also P. Waentig, 2. physik. Chem., 68, 513 (1909) and J. H. Hildebrand, ibid., 74, 679 (r9ro). 
the solubility in benzene would be represented by a line parallel and close to the line for chloroform.

The same behavior is shown by carbon disulfide solutions, which begin to tum brown below $0^{\circ}$, indicating solvation and producing a higher solubility than would otherwise be found. The dotted lower extension of the curve for this solution shows approximately the course the curve would take in the absence of solvation. We wish to emphasize that it is the deviation of the actual curve from this dotted curve, and not from the line of the ideal solution higher up which should be used for any estimate of the amount of solvation. The well-nigh universal practice of concluding that all deviations from Raoult's law, or, still worse, from van't Hoff's law for osmotic pressure or from any of the laws derived from them, indicate changes in the molecular species present in the solution, is far from being justifiable, as the present illustration clearly shows. The considerable deviation of solutions of iodine in heptane from Raoult's law that would be shown by molecular weight determinations as well as by the solubility curve, might be "explained" by assuming that iodine is "associated" in. heptane, or, if one prefers, that heptane is associated-either will work. However, either is unjustifiable, for there is no more effect of this sort in heptane that there is in carbon disulfide.

The above results are at most but slightly influenced by any tendency of benzene to form solid solutions in iodine. A solution of iodine in benzene was slowly evaporated at approximately $45^{\circ}$ and the iodine crystals remaining were analyzed. They were found to contain $98.7 \%$ iodine. Since some benzene may have been occluded, the amount in solid solution must have been very small. Evidently the solubility of benzene in solid iodine is much less than that of iodine in benzene.

The curves in Fig. 3 would enable one to construct the whole solubility curve for any solvent for iodine which gives violet solutions provided a single point is known. Thus the solubility of iodine in hexane has been determined at $5^{\circ}$ by $\mathrm{E}$. 'T. Ellefson in this laboratory, ' giving $N=0.0045$. a value a little less than the corresponding one for heptane. A curve drawn through this point parallel to the curve for heptane would undoubtedly give very closely the solubility in hexane through the corresponding range of temperature. Jakowkin ${ }^{2}$ gives a value for the solubility of iodine in bromoform at $25^{\circ}$. Unfortunately, it is not clear whether his figures tefer to amount of iodine per liter of solvent or of solution. Since the solubility is large it makes a considerable difference which is used in calculating to mol. fraction. If the former is correct $N=0.062$, while if the latter is correct, and assuming that the volume of the solution is the sum of the volumes of bromoform and liquid iodine, $N=0.066$. In

1 E. T. Ellefson, Thrs Journat, 39, з301 (I917).

${ }^{2}$ Jakowkin, Loc. cit. 
either case the solubility is greater than that in carbon disulfide, in accord with the greater internal pressure of bromoform, and the entire solubility curve could be drawn parallel to that for carbon disulfide.

If we consider a solvent for which we have no solubility data, such as ethylene bromide, we can predict the solubility with a fair degree of approximation by noting that this solvent has an internal pressure a little less than that of carbon disulfide, hence its solvent power for iodine should also be a little less, and its solubility curve should be drawn a little below that for carbon disulfide and parallel to it, since it gives violet solutions showing no solvation.

When polar solvents are used, or those giving brown solutions, the solubility cturve will be more unique, behaving like the benzene curve but in a more exaggerated form, and in a position more difficult to predict.

\section{Summary.}

1. A method for criticising solubility data has been outlined, according to which the solubilities of a substance in solvents where no molecular changes are involved, when plotted as $\log N$ against $\mathrm{I} / T$, give a family of approximately straight lines converging to the melting point of the solute where $N=\mathrm{r}$. The position of these lines corresponds to the internal pressures of the solvents and is approximately predictable from them.

The effect of solvation upon the solubility and also upon its temperature coefficient has been outlined.

2. The above mentioned has been illustrated by means of solubility data of naphthalene in various solvents.

3. Solubilities of iodine in benzene, carbon tetrachloride and heptane have been measured at various temperatures.

4. The solubility relations of jodine have been discussed by the aid of the above method, and it is shown that the violet solutions give the expected family of paraliel curves, whereas the brown solutions show deviations in the direction expected.

BaMEEIEY, CAT. 